\title{
Italique Italique
}

Poésie italienne de la Renaissance

XIII | 2010

Varia

\section{Il Negromante de l'Arioste traduit par Jean de La Taille}

\section{Riccardo Benedettini}

\section{(2) OpenEdition}

1 Journals

Édition électronique

URL : https://journals.openedition.org/italique/284

DOI : $10.4000 /$ italique.284

ISSN : 1663-4438

\section{Éditeur}

Librairie Droz

\section{Édition imprimée}

Date de publication : 1 décembre 2010

Pagination : 81-104

ISBN : 978-2-600-01489-2

ISSN : $1423-3983$

\section{Référence électronique}

Riccardo Benedettini, « II Negromante de I'Arioste traduit par Jean de La Taille », Italique [En ligne], XIII I 2010, mis en ligne le 23 juin 2014, consulté le 21 septembre 2021. URL : http://

journals.openedition.org/italique/284; DOI : https://doi.org/10.4000/italique.284

(c) Tous droits réservés 


$$
\text { RICCARDO BENEDETTINI }
$$

\section{IL NEGROMANTE DE L'A R I OSTE T R A D U I T P A R JEA D E L TAILLE}





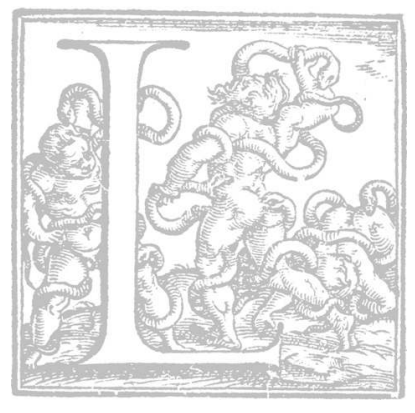

a traduction française du Negromante de l'Arioste par Jean de La Taille a probablement été composée vers Is 62. ${ }^{\mathrm{I}}$ Publiée à Paris chez. Federic Morel «Imprimeur du Roy», en I573, dans un volume qui réunit plusieurs autres cuvres poétiques du seigneur de Bondaroy, ${ }^{2}$ la comédie passe des vers italiens à la prose française. Comme plusieurs critiques l'ont déjà signalé, il est significatif que le texte de Jean de La Taille ait suivi la première rédaction de la pièce de l'Arioste, celle de I520 (son unique manuscrit, le «Di casa Minutoli Tegrimi» de Lucques, fut acheté par Michele Catalano à Florence en 1925). Cette première édition établie par Lodovico Dolce et publiée en I535 chez. Francesco Bindoni et Maffeo Pasini à Venise, est en effet moins élaborée que la rédaction défnitive, publiée chez Gabriel Giolito de' Ferrari à Venise en I55I. ${ }^{4}$ Comment de La Taille a-t-il exécuté la traduction de cet ouvrage? Parmi les nombreuses éditions de I535, possédait-il, comme on l'a dit, un exemplaire du Negromante publié par Zoppino à Venise? Peut-être. Il est sans doute difficile de le prouver matériellement à partir de quelques différences textuelles, même si celles-ci sont plus nombreuses et parfois plus singulières que celles qui caractérisent les autres réimpressions. ${ }^{5}$ Dans la mesure où des leçons propres à cette édition different des textes plus corrects, en modifiant par conséquent le discours français, nous en signalons les variantes; ce décalage dans les éditions indique que, même si un grand nombre d'interventions dans la traduction est supposé être influencé par $Z$., nous avons toutefois des leçons qui en divergent et qui nous semblent montrer la nécessité d'une identification des phases différentes de la tradition textuelle de cette cuvre. Dans l'impossibilité de bien identifier le texte dont le traducteur s'était servi, il faut se demander si La Taille a pu travailler sur deux éditions différentes: le recours à l'édition $Z$. et à l'édition de ISII permet de suivre l'élaboration de la traduction dans ses différentes phases. Et là on voit combien, en établissant un parallèle entre la fidélité de la traduction de Jean de La Taille d'une part, et d'autre part le caractère particulièrement incorrect de l'exemplaire Zoppino, nous pouvons caractériser cette traduction comme un exemple de ce que l'on pourrait appeler une belle "fidèle infidèle».

Une fois ce problème des sources indiqué, on en sait un peu plus sur une traduction qui, selon les termes de Cioranescu, semblait s'écarter souvent de l'original, avec la suppression de passages entiers, la création de scènes qui ne se trouvaient pas dans le texte de l'Arioste, bref «avec des modifications dont on ne comprend pas trop bien le buts. ${ }^{6}$ En revanche, la comédie en prose de Jean de La Taille, qui garde aussi bien le prologue que le fractionnement en 
scènes et en cinq actes, ne propose aucune rupture radicale avec la rédaction italienne de I520: ces écarts, dont a parlé la critique, ne sont que des variantes imputables à l'Arioste, qui a écrit deux versions profondément différentes de la même cuvre, dont le seul aspect qui ne change pas est le vers. Dans un premier temps on devine donc une traduction presque littérale, qui respecte le niveau linguistique, artistique et structural de cette première élaboration textuelle. ${ }^{7}$ Mais une analyse attentive de la pièce française permettra de relever des variantes synchroniques, souvent minimales, qu'on doit en revanche attribuer et au traducteur et aux fautes d'impression de l'édition utilisée. Dans cet apparat, les variantes structurales constituent les éléments d'une corruption paléographique reconnaissable sur la charpente du texte, des erreurs (in)volontaires qui nous semblent aussi bien le résultat d'une mauvaise lecture de l'original que la conséquence des erreurs considérables qui investissent le texte de départ. Nous avons ensuite des variantes d'ordre linguistique, en particulier de pertinence lexicale, qui sont le témoignage de conjectures interprétatives du traducteur. Que Jean de La Taille connaisse bien l'italien cela va de soi. Qu'il arrive à le maîtriser, on le voit dans cette traduction, dont il conserve en général le discours construit par l'auteur, gardant même les non-sens présents dans son édition de référence: la traduction suit exactement le texte italien et s'attache à en rendre chaque terme avec précision. Mais dans cette compétence il faudra cependant tenir compte de certains manques de comprébension et de difficultés de connotations textuelles que La Taille transcrit souvent en marge de son Negromant et qu'il résout selon un processus partiellement cohérent: c'est le sens de l'ensemble qui le guide et le préoccupe. ${ }^{8}$ À côté des observations sur l'action, chargées d'importance pour l'élaboration d'un travail sur un texte théatral, on ne peut donc se contenter de reconnaître et d'identifier les liens qui rattachent la parole à l'action, mais on doit pousser plus loin l'analyse de la langue pour voir si l'xuvre de Jean de La Taille est le reflet d'une perméabilité entre la langue française et la langue italienne, perméabilité qui suppose une convergence de gô̂t ainsi qu'une affinité culturelle, appartenant, comme nous le savons, aux nombreuses traductions de l'italien réalisées au cours du

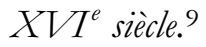

Pour comprendre l'attitude de Jean de La Taille, il faut partir du point de départ le plus évident, à savoir le prologue de l'édition italienne. Si la version en prose des 54 vers de l'Arioste (68 vers dans l'édition de I5II) marque une première étape dans la transposition ponctuelle en langue française, il importe pour nous que de La Taille ait conservé la formule du «Prologue» (Z., 99vIoor) où un personnage tient un discours en aparte. ${ }^{10}$ Nous lisons dans les premières lignes de ce texte: "Ne pensez plus ouir chose impossible, si on vous dit que les cailloux, et les arbres, de contree en contree suivoyent Orfee») $(Z$., 99v). On pourrait regretter de se trouver face à un cortège de «cailloux» 
alors que, sur la base traditionnelle du récit ovidien, c'est un «ferarum / concilio» (Métamorphoses, X, 143-44) qui assiste le poète: la discontinuité $d u$ topos n'est pas un choix subjectif $d u$ traducteur mais une variante d'auteur, qui écrit «i sassi» en Is20 et change son texte en «le fiere» dans l'édition de I5SI. ${ }^{\text {II }}$ Nous dirons donc qu'il n'existe presqu'aucun endroit dans cette comédie qui ne montre la volonté propre à l'Arioste de corriger, de changer, bref de procéder à un grand coup de lime. Dans cette voie, on s'aperçoit que de nombreuses variantes attribuées au traducteur constituent le résultat de sa volonté d'adhésion au(x) texte(s) dont il dispose. Il faut donc distinguer entre des variantes d'ordre textuel (entre les différentes éditions du texte de l'Arioste) et les écarts, ou différences, entre le texte original italien et la traduction française: celles-ci peuvent être volontaires ou le fruit de simples erreurs.

L'adoption du modèle italien apparaît aussi dans le respect, tout en les francisant, du nom du lieu où se passe la scène et celui des quatorze personnages, protagonistes dont les noms italiens se renouvellent d'une édition à l'autre. ${ }^{12}$ Mais si dans la traduction de la pièce nous trouvons un grand contraste en ce qui concerne les marques de politesse proprement dites, c'est-àdire la variation entre le constant tutoiement de l'italien et le fréquent vouvoiement du français (l'alternance entre le tu et le vous est insérée de temps en temps en français, mais seulement si elle correspond à une différence sociale), ${ }^{13}$ dans l'ensemble, les échanges entre les interlocuteurs suivent le texte de l'Arioste, mis à part des changements qu'il faut voir d'un peu plus près dans la mesure où ils marquent une adhésion à l'exemplaire Zoppino. On remarque qu'aucun des cinq actes n'affiche un plus grand nombre de déplacements. Ces écarts se ramènent à deux types: d'un côté des altérations dans l'échange des répliques, de l'autre des coupures qui effacent des éléments du texte. ${ }^{\mathrm{I}}$ Dans ces variations, qui doublent (ou partagent en deux) la portée des dialogues et qui présentent des discours différant de l'original, tout en restant fidèles à l'édition de référence, se manifeste souvent très clairement une réduction de la valeur plaisante de certaines affirmations, confiée dans le texte italien non seulement à l'écrit, mais aux répliques rapides des acteurs, qui accompagnaient évidemment leur voix avec la gestualité et la mimique. ${ }^{\mathrm{IS}}$

La deuxième scène de l'acte premier représente le dialogue entre les deux vieillards Lippe et Cambien: le souvenir de la vie commune passée à Florence ("Firenze») devient chez La Taille «Firense», décalage morphologique qui manifeste peut-être la volonté de rendre graphiquement la plus douce prononciation française du fricatif italien ' $z$ ) aboutit au temps présent à Crémone (Cremona, en italien). Lorsque le florentin Lippe entend le revenu que Cambien obtient en toute saison de sa jeune fille "gentille, gracieuse et belle», il est surpris du changement d'attitude de son ancien concitoyen: 
Lippo. Ah Cambio, ma l'onore ? Cambio. Non sono simili / cose vergogna qui. Quanti ne credi tu, / che sien in questa terra, che più tengono / per uso altrui le moglie che pel proprio ? / E di qui vanno ben vestiti e pasconsi / come abbati e disagio alcun non sentono ? [Lippo.] Or questa si può ben nomar republica! (G., I, 2);

Lip. Ah Cambio, ma l'honor ? Là non son simili / Cose a vergogna. CAM. Qui quanti ne creditù, / Che siano in questa terra; che piu tengono / Per uso altrui le mogli, ch'e pel proprio; [...] Hor questa si puo ben chiamar Republica. (Z., 4v-5r) $\rightarrow$

Lippe. Há combien, mais l'honneur. Ne sont-ce pas choses, dont on deust avoir vergongne ? CAMBIEN. Combien pensez vous qu'il y en a en ce païs, qui tiennent des femmes plus pour l'usage d'autruy, que pour le leur propre? Et mesmes de ceux qui vont bien vestus, et pense bien qu'ils n'en ont point de fascherie, ny de mal-aise. Or cecy se peut bien nommer Republique. (I03V-IO4r)

L'exclamation concernant la singulière république change donc de locuteur; s'il est vrai qu'on garde cependant la valeur ironique de l'information, c'est la stichomythie, cet effet saccadé obtenu par la scansion rapide des vers, qui procède à un changement, que l'on pourrait peut-être en même temps identifier dans la gestuelle des acteurs. ${ }^{16}$ Ainsi le début de la scène quatre, qui commence sur une double réplique, Maximo. Cintio. / Cintio. Messere. / Maximo. Odimi un poco $(G ., I, 4)$, alors qu'en français on ne retrouve que l'appel de Cinthien. Monsieur. Maxime. Escoute un peu (IIor), traduction fidèle de «CYN. Messere. MAs. Odimmi un poco» (Z., Ior). Le passage suivant, tiré du quatrième acte, présente une situation parallèle. La conversation entre Cinthien et Cambien est remplacée en français par une brève réplique du premier personnage, changement dont l'origine se trouve sans doute chez $Z$. et qui implique une adaptation de la ponctuation du texte:

Cintio. Son iti insieme a Maximo ? [CAmbio. Sì, sono. Cintio.] Io son spacciato, io son morto! Apriti, apriti, per Dio, terra, e sepelliscimi (G., IV, 7);

Cyn. Son iti insieme a Massimo / In tutto io son spacciato. Io son morto. Apriti / Apriti per Dio Terra, e seppellissemi (Z., 3or) $\rightarrow$

Cinthien. Ils sont allez ensemble vers Maxime. Ie suis totalement despeché, ie suis mort. Ouvre, ouvre toy Terre, et m'ensevely. (1 $36 \mathrm{v}$ )

L'altération du résultat français voit ensuite l'adjonction de quelques mots prononcés par Cinthien, [...] ie m'en vas (I36v), qui remplace l'impératif de l'original italien, CAмвіо. Aspetta $(G ., I V, 7)$, version parallèle à $Z$. qui présente la formule «CYN. Io vado» (zor) mais où manque l'impératif prononcé par Cambio. Il en est de même pour l'élimination d'une constatation de Themole pendant un dialogue avec Cambien au cours du troisième acte: 
CAmbio. [...] Or accadendoci / questo bisogno, muterà proposito, / e tanto ne farà impegnare e vendere, / che cinquanta fiorin ne trarrà subito. [TEmolo.] Come vien bene a taglio (G., III, I);

CAm. Hora acadendomi / Questo bisogno; mutera proposito / E tanto ne fara impegnar e vendere; Che cinquanta fiorin ne trarrà subito, / Come vien ben in taglio (Z., I 8r) $\rightarrow$

CAmbien. Ores m'estant escheu ce besoing, ie changeray d'intention, et en feray tant engager et vendre, que i'en tireray bien tost cinquante florins, comme cela vient bien à point. (I 20V-2 I r)

Il est évident que ces passages des répliques rapides aux courts monologues ne pèsent pas sur le développement de l'action, mais ils en modifient son commentaire. Ces altérations comportent aussi l'abandon de la transition régulière entre deux scènes du même acte. On fait allusion à l'acte IV, au moment du passage entre la fin de la sixième scène et le début de la septième:

Cambio. Aspetta, aspetta, che fuor esce Cintio (G., IV, 6);

TH. Aspetta aspetta: che fuor esce Cynthio; (Z., 29r) $\rightarrow$

Themole. Attendez attendez: voicy Cinthien qui sort. (1 $35 \mathrm{v}$ )

A remarquer, le changement des locuteurs. Une évolution semblable se vérifie à la fin de la comédie. ${ }^{17}$ D'une manière inattendue et, du moins apparemment, sans une véritable explication, La Taille interrompt un dialogue de la troisième scène pour en créer une quatrième qui ne change rien à l'action:

Camillo. [...] Hai tu trovato, Maximo, / ch'io sia bugiardo ? Maximo. Non, per Dio, ma ascoltami. (G., V, 3);

Cam. [...] Ritrovato hai Massimo, / Ch'io sia buggiardo. Mas. Non per Dio. Ascoltami. (Z., 32r) $\rightarrow$

Camille. [...] Avez vous retrouvé, Maxime, que ie soy menteur ? Scene III. Maxime. Non par Dieu. Escoutez moy. (I 39r);

De même, une omission, une insertion et un changement de la disposition des répliques caractérisent le dialogue entre Maxime et Camille sur le thème du mariage, mais ces variations sont cette fois encore en accord avec la version de $Z$.:

Maximo. Dio conduca e prosperi, / senza mai lite averci, il matrimonio. / [Sian d'accordo ?] Abondio. D'accordo. Camillo. D'accordissimo (G., V, 3);

Mas. Dio conduca, e prosperi / Senza mai lite haverci, il matrimonio. CAm. Siam d'acordo. Ав. Dacordo. Cam. D'acordissimo (Z., 33r) $\rightarrow$ 


\section{RicCARDO BENEDETTINI}

Maxime. Dieu conduise et prospere le mariage sans iamais avoir noise. Camille. Nous en sommes d'accord. Abonde. D'accord. Maxime. Plus que d'accord. (140r)

Les derniers vers de la comédie accentuent l'importance de ces écarts documentés au niveau du dialogue. Si chez. l'Arioste c'est le personnage de Camillo qui prononce les derniers mots, chez La Taille ce sera Maxime, à cause de la mauvaise distribution des répliques qui se trouve dans le texte de départ:

Camillo. E perché non concludere / presto quel che s'ha a far? / Maximo. Ben dice, sposila / ora. / Abondio. Sposila, andiamo. / Camillo. Andian, di grazia. Non aspettate, olà! che torni Cintio, / che per l'uscio di drieto è intrato tacito / in casa. E chi del negromante intendere / vuole, gli corra drieto; ma spediscasi, / ch'el va che par che se lo porti il diavolo. / A Dio, benigni guardatori. Fatene / con alcun segno d'allegrezza intendere / che piaciuta vi sia la nostra fabula. (G., V, 3);

CAм. E perche non concludere / Presto quel, che s'ha a far. Mas. Ben dice, sposila / Hora. Ав. Sposila, andiamo. Cam. Andiam di gratia. / Mas. Non aspettate ò la: che torni Cynthio, / Che per l'uscio di drieto è intrato tacito / In casa. E chi del Negromante intender / Vuole; gli corra drieto: ma spediscasi: che va, che par, che se lo porti il Diavolo. / A Dio benigni guardatori. Fatene / Con alcun segno d'allegrezza intendere, / Che piaciuta vi sia la nostra fabula. (Z., $35 \mathrm{r}) \rightarrow$

Camille. Et pourquoy ne concluez-vous viste ce qu'on a affaire ? Maxime. Il dit bien, qu'il espouse sur l'heure. Abonde. Qu'il espouse, allons. Camille. Allons ie vous prie. Maxime. N'attendez point là que Cinthien retourne, car il est entré secrettement par l'huis de derriere en la maison. Et qui voudra entendre du Negromant, s'y courre apres, mais qu'il se despeche, car il va si fort qu'il semble que le diable l'emporte. A Dieu benins spectateurs, et avec aucun signe d'allegresse faites entendre que nostre fable vous a pleu. (I42r)

À ces différentes répartitions sont associées des coupures qui privent le texte d'indications qui n'ont pas toujours la valeur d'un détail. Dans le troisième acte, le paiement demandé par le Fizicien à son client disparaît:

Fisico. [...] che dimandato ho il vitel per mangiarlomi. Maximo. Chi ha così detto ? [Fisico. E i fiaschi per rubarteli.] Maximo. Chi ha detto cotesto ? [...] Fisico. [...] e per che veggino / ch'io non dimando 'l vitel per mangiarlomi, / voglio in casa tua fare il sacrifizio, / [nel qual sei tazze, tondi, fiaschi e simili] / cose vorrò, che molte ci bisognano / oltre a quelle c'ho dette, non levandole / di casa tua (G., III, 4); Fis. Che dimandato ho il Vitel per mangiarlomi. / Mas. Chi ha cosi detto. Fi. E i fiaschi per rubarteli. / MAs. Chi ha detto cotesto. [...] F. [...] e perche vegghino, / Ch'io non dimando il Vitel per mangiarlomi, / Voglio in casa tua far il sacrificio. 
Cose vorro (Che molte ci bisognano) / Oltre queste c'ho detto: e non levandole / Di casa tua; non diran quel, che dicono (Z., 23r) $\rightarrow$

Fizicien. que i'avois demandé le veau pour le manger. MAXime. Qui a dit cela ? [...] Fizicien. [...] et à fin qu'ils voyent que ie ne demande pas le veau pour le manger, ie veux faire le sacrifice en vostre maison. Ie voudroy des choses (car i'en ay besoing de beaucoup) outre celles que ie vous ay dittes, et ne les ostant point de vostre maison. (I $26 \mathrm{v}-27 \mathrm{v}$ )

Dans la scène quatre du premier acte la section finale du dialogue entre Maxime et le jeune Cinthien est absente:

Maximo. Ma or ch'hai moglie allato, e che i tuoi suoceri / si son doluti meco di tal pratica, / [et han sospetto che questa sua femina / non t'abbia così concio, voglio rompere / questo silenzio, e dirti che malissima- /mente fai, più tenendo cotal pratica] (G., I, 4);

Mas. Ma hor c'hai moglie al lato; e ch'i tuoi soceri / Si son doluti meco di tal pratica (Z., Ior) $\rightarrow$

MAxime. [...] mais ores que tu as femme à ton costé, et que les parens d'icelle se sont plaints à moi de telle pratique. ( I I ov).

Le point final qui a été introduit dans cette version implique une diminution de la vivacité du texte. ${ }^{18}$ L'acte II confirme ces idées. L'échange des répliques entre Camille et le Fizicien devient en revanche plus rapide chez La Taille qui, à l'instar de $Z$., coupe en deux l'observation du jeune amoureux en élargissant le rôle du Negromant:

Camillo. Te ne supplico. / Di quelle man, più che di latte candide, / più che di neve, è uscita questa lettera (G., II, 4);

CAm. Te ne supplico / Fi. Di quelle mani più che latte candide; / Piu che di neve, è uscita questa lettera. (Z., IGr) $\rightarrow$

Camille. Ie vous en supplie. Fizicien. De celles mains plus blanches que lait, et que neige, est sortie ceste lettre. (1 I $8 \mathrm{v}$ ).

L'omission modifie en revanche la conclusion de cet acte. Si l'Arioste exprime d'une manière nette le désaccord entre la Fantesque et la Mère à propos du malentendu sexuel qui est au centre de l'action tout entière, la version utilisée par le traducteur donne au texte un caractère plus tempéré, qui ne rend pas compte de la portée ironiquement obscène du texte le plus diffusé:

Fantesca. Dunque, mi debb'io vergognare a dirte la / veritade? [S'ancor tu experienzia / fatta avevi di Cintio, a questo termine / non saresti ove sei. Ma che più ? Reputa / che sia tutto uno, poi che experienzia / n'ha fatto tanti dì tua figlia, 
lascialo / con sua mala ventura, e d'altro genero / ti provedi. Ma fa a mio senno: pruovalo / tu prima. MAdre. O sie in malora! Che consiglio / mi dà questa gaglioffa! Fantesca. Se tu prendere / questo non vuoi, te ne do un altro.] Lascialo / provare a me. S'io il pruovo, far iudicio / saprò s'accontentar ne avrà Emilia. MADRE. O brutta, disonesta e trista femina, / serra la bocca in tuo mal punto, e seguimi (G., II, 5);

FAn. Dunque io mi debbo vergognar a dirtene / La verita; s'anchora a la esperientia / Ne ha fatto tanti di tua figlia; lascialo / Provar a me, s'io il provo far giudicio / Sapro, s'accontentar se ne hara Emilia. / MAD. O brutta dishonesta e trista femina / Sera la bocca un tuo mal punto e seguimi (Z., I $7 \mathrm{v}) \rightarrow$

LA FANTESQUe. Doy-ie donc estre honteuse à vous dire la verité ? Que s'il n'a fait encor telle experience de vostre fille, laissez-le essayer à moy: si ie l'essaye ie sçauray bien iuger si Emilie s'en devra contenter. La Mere. O vilaine, deshoneste et meschante femme, serre la bouche à la malheure, et me suy. (I 2or)

La mise en français de l'opposition entre ces deux attitudes féminines dépasse le niveau d'écart involontaire et nous pousse à rechercher des motifs de détail et de style. C'est là un aspect fondamental dans le passage de l'italien au français qui sollicite les traducteurs du XVI ${ }^{e}$ siècle. Le rapport entre la langue et la culture peut s'expliquer avec la substitution d'un motpar un autre, c'est-à-dire par une intervention qui résout le conflit, tout en dénonçant le changement de perspective culturelle: voilà qu'il prend au sérieux la sexualité concrète ou abstraite; voilà qu'il intervient sur le plan des fonctions expressives et des choix lexicaux, stylistiques et grammaticaux du texte, en particulier sur le plan des figures rhétoriques. En ce sens, le traducteur peut choisir de s'enfermer passivement dans la plate transposition des mots, apportant au public son expérience textuelle, ou bien il propose ce qui est dissimulé derrière les mots, conscient des connaissances et des maurs qui sont propres aux hommes de son temps et de sa société. Cette seconde perspective, qui témoigne de la volonté d'aborder un style culturel commun, concerne d'abord les expressions figurées et la tentative de retrouver en français une parfaite équivalence sémantique des séquences textuelles sans compromettre le modèle. Chez.Jean de La Taille, la traduction naît du respect du ton de l'Arioste qui, dans Il Negromante, se plaît à utiliser un langage familier, populaire, proche du jargon, avec des nuances comiques voire satiriques: c'est pourquoi il a recours au toscan, dialecte littéraire, fait d'à-peu-près, écrit par un Émilien, et qui atteint directement la sensibilité des lecteurs ou des auditeurs italiens par sa tendance à la représentation matérielle des faits, aspect qui se trouve évidemment affaibli dans la version française. À cet égard, il convient de considérer les changements opérés dans cette transposition de certaines expressions au ton souvent cru. Populaire, violent, très explicite et sincère, c'est là le soubait prononcé par la chambrière Aurélie pendant son dialogue avec Marguerite: Aurelia. Che 
nasca una fistola / a chi mai fece questo sponsalizio! (G., $I, I ; Z$., 3r) $\rightarrow$ Aurelie. Que mal advienne à quiconque feit iamais ces espousailles! (IOIV) Le traducteur garde la réplique mais d'une manière bien allégée, qui prive son texte de l'invitation perverse au rire. ${ }^{19}$ La description successive de l'échec du jeu amoureux garde dans notre édition la valeur renforcée de l'italien: AureLia. Deh, non temer che giostrino, / che la lancia è spuntata e molto debole (G., I, I; Deh non temer, giostrino $[\ldots], Z ., 3 v) \rightarrow$ Aurelie. Deà ne crain point qu'ils ioustent: car la lance est epointée et trop foyble (IOIv). ${ }^{20}$ C'est la (fausse) impuissance sexuelle de Cinthien, information à la base de l'élaboration de cette comédie, qui fait parler ici les personnages. Pour traduire le mot impotenzia (et son dérivé adjectival impotente), La Taille utilise les mots «impotence» (III, 3, I24v), «debilité et impotence» (II, 3, II rr), «infirmité» (II, 4, II 8r), la forme plus discrète d'«indisposition» (I, 3, I08v), tout comme ces adjectifs: «impotent» (I, 2, I06v; III, 2, I2Ir), «indispost et debile» (I, 2, I07r), «[ny] malade ny debile» (II, I, II Jv), «debiles» (I, I, I02r), «impotent et debile» (II, 3, II $6 r) .{ }^{2 I}$ On retrouve ce même style quand il s'agit des conséquences liées à cette «faiblesse» (l'italien debiltade, G., II, 3, est rendu par «debilité et impotence», IIGr): le repudio (G., III, 2), qui devient le «divorce» (I22r). Au deuxième acte, on assiste à un dialogue entre la mère et une «fantesque». Celle-ci soutient encore "qu'on ne devroit point prendre les marits sans les essayer》 (IIgv); voilà que la mère lui répond: «Taci, porca, e vergognati» (G., II, 5; Z., I7v) $\rightarrow$ «Tay toy, vilaine, as tu point de honte ?» (I2or). Le niveau lexical a beaucoup changé, et l'interrogation a réduit le caractère incisif de l'impératif. Cet allégement du côté charnel du texte se retrouve dans la restitution des paroles du physicien quand il montre son travail à Cintbien: Fisico. [...] ogni opera / mia è però fin qui stata favorevole, / più presto alle tue voglie, che contraria. (G., III, 2); ogn'opera / Mia è però fin qui stata favorevole / Assai piu alla tua voglia, che contraria $(Z$., $I 8 v) \rightarrow$ LE Fizicien. [...] tout ce que i'ay fait a esté assez plus favorable à vostre souhait qu'autrement (I2Ir).

D'autres expressions manifestent ces différences de niveau linguistique. En aparté, Cinthien rappelle la récompense que le Negromant lui a demandée pour son travail: «con sue lusinghe» (G., III, 2) $\rightarrow$ «par ses paroles» (I23v); il explique l'augmentation du prix à payer: ${ }^{22}$ d'ailleurs, nous savions que le Fiqicien voulait «porre in un cadavere / un spirto» $(G ., I, 3) \rightarrow$ «mettre un esprit en un corps mort» (I08v), et qu'il avait déjà reçu «un bellissimo / rubin» (G., II, 3; Z., Isr), pierre dont la beauté est exprimée avec le très italien superlatif en -issimo qui se perd dans ce passage au français, «un beau rubis» (II7r). Ce même processus de transposition conduit à charger les 


\section{RicCARDO BENEDETTINI}

métaphores d'une réalité plus concrète, impliquant la perte ou la réduction de cette figure:

Lippo. [...] con quanta fretta gli anni volano! (G., I, 2) $\rightarrow$ Lippe. [...] comme les ans vont viste (I04v);

Cambio. Perché da me sì subito / s'è dileguato ? (G., IV, i) $\rightarrow$ Cambien. Pourquoy s'est-il party de moy si tost? (I zov);

Cambio. [...] accesesi (G., I, 2) $\rightarrow$ Cambien. [...] il s'enamoura (Io5v);

Abondio. [...] non avete rossor (G., V, 2) $\rightarrow$ Abonde. [...] n'avez vous point de honte (I $38 \mathrm{r})$;

Temolo. [...] non c'è quel pericolo / che le hai dipinto (G., IV, I) $\rightarrow$ Themole. [...] il n'y a point icy de danger, comme vous luy aviez dit (I zor);

Temolo. [...] arreca li denari e fagline / pala (G., III, I) $\rightarrow$ Themole. [...] aprestez les deniers, et faittes paction avec luy ( $\mathrm{I} 2 \mathrm{OV}$ );

Chez La Taille nous rencontrons aussi des omissions qui privent le texte français du caractère accentué par rapport à la norme italienne, selon des critères qui ne correspondent pas toujours au texte de Z.; le traducteur omet les 'excès' d'adjectifs, certains détails descriptifs, la duplication tautologique des verbes, mais aussi certaines répétitions qui auraient davantage conservé le ton parlé de l'italien:

per difficile / strada, [piena di fanghi] e di monti asperi (G., vers I 3-I 4 du Prologue; Z., ivi) $\rightarrow$ par un chemin facheus et plein d'aspres montaignes (99v);

de l'[alta] cortesia (G., v. 27; Z., ivi) $\rightarrow$ de la courtoisie (Ioor);

de la [somma] virtù (G., v. 28); De la virtute (Z.) $\rightarrow$ de la vertu (Ioor);

un vitel [nero], ma di latte e morbido (G., I, $3 ;$ Z., $8 v$ ) $\rightarrow$ un veau, mais qu'il fust de lait et gras (108v);

un poco nero [e grasso] (G., I, 3); un poco nero (Z., 8v) $\rightarrow$ «un peu noir» (Io8v); Anch'io talora muovola [la terre] / s'io metto a fuoco [o ne lievo] la pentola; / [o quando al buio sento se più gocciola / di vino è nel boccale,] alor dimenola (G., II, I); Th. Anch'io tal'hora muovola; / S'io metto al fuoco, o ne levo la pentola (I Ir) $\rightarrow$ Ie la remeus telle fois moy mesmes, si ie mets un pot au feu, où si ie l'en oste (I I $2 \mathrm{r}$ );

[aggira et aviluppa] (G., II, 2; Z., I 3r) $\rightarrow$ il envelope (I I 4v);

che tu la mia prospera / fortuna sei, salute, [vita] et anima (G., II, 4; Z., I sv) $\rightarrow$ car vous estes ma bonne fortune, mon salut, et mon ame (i I $7 \mathrm{v}$ );

certissima- / mente ti fo saper che la tua Emilia / è in tal voglia ...; [che voglia ? è] in tal rabbia / d'esser teco (G., III, 3); certissima-/ Mento ti fo a saper, che la tua Emilia / E in tal voglia, che voglia: è in tal rabbia / d'esser teco» (Z., 20v) $\rightarrow$ ie vous fais à sçavoir pour certain que vostre Emilie a telle envie et telle rage d'estre avecques vous (I 24r); 
'l maggior scandolo, / [il maggior scorno, il maggior vituperio] (G., III, 3); che saria il maggior scandolo (Z., 2ov) $\rightarrow$ le plus grand scandale (I 24r).

À côté de ces omissions et de ces figures, plus ou moins traditionnelles, notons les témoignages métaphoriques qui comportent des allusions sexuelles et qui sont encore plus difficiles à situer. Avant d'aborder les écarts de traduction, nous voulons rappeler un exemple de correspondance formelle et sémantique entre le terme italien et son équivalent français, qui est bien résolu par La Taille. L'un des pouvoirs magiques du Fizicien est de transformer un bomme en animal; dans une métaphore filée, les animaux concernés sont le loup, le renard, le milan, l'âne et finalement le bouc: Temolo. Di molti, che diventano / becchi, mi vo' tacer (G., II, I) $\rightarrow$ Themole. Ie ne dy mot de beaucoup qui deviennent boucs (II2v). Le mot becco (au pluriel becchi), qui est ici correctement rendu par «bouc(s)», représente certes en italien le bouc, il capro, il caprone, mais il renvoie très souvent au sens figuré d'homme trahi par sa femme, le cocu, terme qui était attesté avec cette valeur en français au seizième siècle. ${ }^{23}$ Le traducteur a conservé l'idée et le pouvoir métaphorique du mot, peut-être sans le vouloir. En revanche, si l'Arioste a rassemblé en une belle expression le dédoublement causé par la joie des corps dans l'acte sexuel, «che di sé gli fesse copia» (G., I, 2; Z., 6r), La Taille comprend cette union mais il la dessoude: «à ce qu'elle luy feist part de sa personne» (IOSv). Et si au jargon populaire - que l'on sait figuré et à la fois matériel-, appartient aussi un proverbe, "AuRELLA. Gli anni? Domine! Aspettar deve a pascersi / dunque ella, a bocca aperta, finché creschino / le biade ?» (G., I, I; Z., 3v), bien adapté au public français, "AURELIE. Les Dames doivent elles attendre les ans à se repaistre? Donc elle beante attendra-elle que le pain luy tombe dans la bouche?» (I02r), il est en revanche évident que le substantif "les Dames» ne correspond pas au signifiant du vocatif Domine!, interjection d'origine latine (de dominus) qui est souvent utilisée en italien dans une perspective d'invocation plaisante et dont le signifié est o Signore!, "Seigneur!». ${ }^{24}$

Nous devons nous interroger en même temps sur d'autres variations qui privent le texte français non seulement de l'aspect brillant de l'original, mais aussi du sens précis de ce que l'Arioste voulait dire par ses figures: babbion (G., IV, s; $Z$., 27v), en italien semplicione, credulone, grullo, le «sot», le «simple», est rendu par bien enfant (I33v), sans doute à cause d'une faute d'interprétation graphique avec le terme bambino et sa variante bambinone, qui est une forme augmentative porteuse d'une connotation plaisante, aussi bien qu'affectueuse, encore amplement utilisée; ${ }^{25}$ sur ce même modèle d'hésitation de la graphie, les "gitans» italiens deviennent en français des «sangliers», NebBio. [...] andiamo come zingani / di paese in paese 
(G., II, 2; "Cingheri», Z., I3r $\rightarrow$ NebBIEN. seul [...] Nous allons comme sangliers de païs en païs (II4V), variation de l'expression comparative, employée pour indiquer ceux qui mènent une vie nomade ou ceux qui ont des attitudes vertement décomplexées, qui a probablement été créée sur la base d'une trivialité du texte: «sangliers», portant d'une confusion entre le mot zìngaro (avec sa forme ancienne cìngaro) et le mot zingiaro, ancienne forme dialectale pour cinghiale, «sanglier» (en toscan, cignale, cingiale). ${ }^{26}$ C'est encore une référence à un mammifère artiodactyle qui pose problème au traducteur dans un conseil que le Negromant a donné à son serviteur: FISICO. Fa pur tu il gonzo, e mostrati / di non aver le capre / NebBio. Starò mutolo $(G ., I I, 3)^{27} \rightarrow$ Fizicien. Fay seulement, pren le Gand, et fay semblant de n'avoir point les sonnettes. NebBiE. Ie ne diray mot (II7r); mais nous savons que la traduction française est paradoxalement fidèle à l'infidèle texte de départ: Fr. Fa pur, to il guanto, e mostrati / Di non haver le campan $(Z$., $I 5 r) .{ }^{28}$

D'autres variantes sont à observer: si le syntagme «cogliere / qualche mazzata» (G., III, 3; Z., 2Ir-2IV) indique bien sûr une «récolte», ce n'est pas au sens de «cueillir quelque moisson on fruict» (I24V), mais a colpi di mazza, «à coups de masse»; ;e de La Taille a sans doute pensé aux mots mazzo, covone, c'est-à-dire «bouquet», «gerbe». La volonté de rester fidèle au texte italien se sent dans des exemples dont le résultat dépasse le cadre original: la locution in piazza (G., II, I) est traduite dans sa valeur métonymique au marché (II 4 r), tandis que le groupe de substantifs, «ciance e menzogne» (G., II, 2; "Ciancia, e menzogna», Z., I3r), relatif au physicien, passe à la forme verbale «il deçoit et ment» (II4v), ce qui est correct si nous prenons le verbe «décevoin» dans son sens vieilli de «tromper, séduire par une apparence qui promet plus qu'elle ne donne», bref dans un aspect sémantique qui concerne les menzogne plutôt que les ciance (pluriel de ciancia), mot qui indique un bavardage souvent inutile et vain. ${ }^{30}$ Parallèlement, La Taille omet le terme ciancia dans un passage concernant l'impuissance sexuelle de Cinthien, mais il comprend bien le signifié de la locution utilisée en italien, dar ciance, qui équivant à far promesse vuote, tenere a burla con chiacchiere: Lippo. Cotesto non cred'io, che gli è impossibile; / ma che vi dia la ciancia vo' ben credere. / CAmbio. Non mi dà ciancia, no: siane certissimo $(G ., I, 2 ; Z ., 7 v) \rightarrow$ Lippe. Ie ne croy pas cela, car il est impossible: mais ie croyroy bien qu'il vous abuse. Cambien. Il ne m'abuse point, non, soyez en certain (I06v-I07r).

On trouve aussi des cas, qu'il est raisonnable de supposer être des fautes, sans aucun doute moins graves que les précédentes du point de vue de la signification du texte, et qui seront pour nous un ultérieur témoignage de l'importance de ces 
écarts dans l'analyse des solutions textuelles adoptées dans une traduction. Ainsi, l'opposition entre l'entendu' et le 'dit' est au centre d'une équivoque verbale engendrée par l'altération des réponses, très simples, entre deux personnages: Cambio. [...] Or odi. Lippo. Odo $(G ., I, 2) \rightarrow$ Cambien. [...] Or escoutez. Lippe. Dittes. (Iosr). À la supposition avancée en italien sur une question d'argent, Temolo. Potrà cinquanta fiorini ? (III, I), correspond en français une perte de cette valeur dubitative et le passage à une certitude, presque à un ordre, Themole. Portez cinquante florins (I20v), traduction fidèle de l'incorrect $\mathrm{T}$ H. Porta cinquanta fiorini (Z., I $8 r)$. C'est toujours l'interprétation d'une forme verbale, CAmillo. Gli parlerò chiarissimo e ben sètene / certi $(G ., V, 2)$, dans laquelle sètene n'est qu'une variation du subjonctif siatene, qui donne un résultat bien différent, Camille. Ie luy en parleray voirement, et bien severement ( $I 38 v)$, ce qui est explicable seulement en le rapprochant du différent texte de départ, Cam. Gli parlero chiarissimo, e ben severamente $(Z$., 3Ir-3IV). Cette attention aux variations verbales nous amène à souligner ces changements, surtout fréquents dans le prologue, qui ne modifient pas le sens du verbe mais son aspect temporel. Nous constatons qu'en général ces différences sont à attribuer au traducteur plutôt qu'à une édition italienne non correcte. C'est le cas du passage du présent au futur: se detto vi sarà (G., v. 2 du Prologue; $Z$., ivi) $\rightarrow$ si on vous dit que (9gv), una comedia / tutta nuova, la qual vuol che si nomini / il Negromante e che oggi a voi si reciti (G., 3 I et s.; $Z$., ivi) $\rightarrow$ une Comedie toute nouvelle, laquelle veut estre nommée LE NEGROMANT, et se iouera enhuy devant vous (Ioor), vivomi / commodamente (G., I, 2; $Z$., $4 v) \rightarrow$ en vivray tousiours commodement (I03v), Vedi trovarne, da chi n'abbia, in prestito (G., I, 3; Vedro trovarne da chi n'habbia in prestito, $Z$., gr) $\rightarrow$ Ie verray si i'en pourray emprunter de quelqu'un qui en aye (Iogr), Temo che mal consiglio dato a Cinzio / avremo (G., III, I; $Z$., $I 7 v) \rightarrow$ Ie crain que nous n'ayons mal conseillé Cinthien (I20v), io non son per dargliene / uno (G., IV, I; Z., 24v) $\rightarrow$ ie ne luy en bailleray pas un (IIgr); du futur au présent: Or non vi parrà più (G., v. 34; $Z$., ivi) $\rightarrow$ Or qu'il ne vous semble plus (Ioor), Ma, se non vi parrà d'udire (G., v. 42; $Z$., ivi) $\rightarrow$ Mais s'il ne vous semble ouir (Ioor), se [...] udirete (G., v. 52-53; $Z$., ivi) $\rightarrow$ si vous oyez (Ioor); et finalement du présent au passé: studia di tradirti $(G ., I V, 7 ; Z$., 39v) $\rightarrow$ il taschoit de vous trahir (I36r).

Nous ne saurions dès lors être surpris de constater que certains noms italiens sont pour de La Taille très difficiles à déchiffrer. Une fois que tous ses crimes ont atteint leur terme, le physicien dévoile à son serviteur son projet final, "ch'avemo a far, se non andarsene / [per Garfignana] in Levante ben carichi?» 
(G., III, s); «c'havemo a far, se non andarsene, / [Perche Carfagna] in Levante ben carichi» $(Z$., 24r) $\rightarrow$ "qu'avons nous à faire sinon de nous en aller en Levant bien chargez? ?» (II $8 v$ ). Garfagnana (aussi bien dans sa variation graphique Garfignana que dans son ancienne forme Carfagna) correspond à un lieu important dans la vie de l'Arioste: c'est dans la ville de Castelnuovo di Garfagnana que l'auteur, avec son fils Virginio, arrivera le 20 février Is22 pour présider le commissariat de la Garfagnana, zone boisée de la Toscane, dans la province de Lucques. ${ }^{31}$ Le toponyme descriptif Castelnuovo fait référence à un lieu avec un château, Castrum Novum, «château neuf» (par rapport à une construction plus ancienne), tandis que la spécification dérive du nom de personne Carfanius ou Carfana avec l'adjonction $d u$ suffixe -ana, d'où Garfagnana. Mais ce nom, cliché narratif d'un lieu isolé et sauvage (on pourrait alléguer l'exemple équivalent de l'Auvergne, en France), choisi non par hasard mais en raison des possibilités que la région offrait à tous ceux qui voulaient se cacher de la justice, est évidemment inconnu au lecteur français, ce qui établit une omission dans le passage vulgarisant d'une langue à l'autre. Il faut d'ailleurs souligner que l'expression Garfignana in Levante devait sans doute être prononcée en accompagnant la voix par des gestes qui rapprochaient les deux toponymes des verbes sgraffignare et levare, synonymes de rubare, «voler» (voir aussi le terme levata, qui signifie rapina, «vol», et fuga, «fuite»). ${ }^{32}$ À l'intérieur de ce passage on note aussi des mutations qui privent le terme italien de sa signification, malgré des formes d'emprunt et de calque qui essaient de conserver l'aspect de la parole, dans la tentative de lui faire posséder approximativement le même sens. L'Arioste, dans son prologue, exprime un jugement linguistique sur les mots utilisés dans cette comédie, dans le cadre d'un changement toscan ("a Fiorenza et a Siena poi diede opera / e per tutta Toscana, all'eleganzia / quanto poté più», G., vs. 47-49) qui peut causer chez. le public (aussi bien auditeur que lecteur) une sorte de bouleversement, «ma se non vi parrà d'udire il proprio / e consueto idioma del suo popolo, avete da pensar ch'alcun vocabolo / passando udì a Bologna, dove è il Studio» (G., v. 42-45; Z., ivr) $\rightarrow$ «Mais s'il ne vous semble ouir le propre et accoustumé langage de son peuple, vous avez à penser que, passant par Boulongne, où il a estudiè» (Ioor). ${ }^{33}$ La traduction que propose La Taille garde le contenu de l'original: "roù il a estudié» se fond harmonieusement dans l'ensemble de la phrase, même si le traducteur a évidemment entendu dove il studiò, avec un passé simple à la place du substantif Studio (en latin Studium, Studium generale), dénotatif qui désignait tout un univers de savoir et de commerce intellectuel dans les villes italiennes du seizième siècle telles que Padoue, la ville universitaire la plus fréquemment citée ou bien, comme ici, celle de Bologne (phonologiquement Boulo(n)gne avec un ou qui est constant). ${ }^{34}$ 
Nous voulons enfin signaler trois exemples qui sont révélateurs d'une difficulté du traducteur à reconnaître l'ambiguité de certains noms quand ils exploitent le langage soi-disant ou ironiquement technique. Themole donne au Nebbie des renseignements pour retrouver le Fizicien:

Temolo. Va dritto fin a un pizicagnolo / truovi sul canto (G., IV, 2);

Тн. Va drieto tu fin in Piugugnolo (Z., 26r) $\rightarrow$

Themole. Va tousiours derriere le Pupagnol iusques au carrefour. (1 3 iv)

Il est évident que La Taille, en cherchant à être fidèle à son texte, crée un mot presque identique à l'original, mais qui n'a pas de sens, et il ne pense pas au terme pizzicagnolo, «charcutier». ${ }^{35}$ Il s'agit d'un calque dans ces deux autres cas. Lippe soutient qu'il est hébergé «da la Semola» (G., I, 2; Z., 8r) $\rightarrow$ «avec ceux du Som» (Io7v); l'élément significatif est ici bien traduit depuis la langue source vers la langue emprunteuse, car le mot italien semola indique exactement la «semoule», le «son». Et de La Taille reconnaît aussi le nom de métier qui était identifié par le -s majuscule: Semola, Son. En revanche, il semble avoir des problèmes quand l'A rioste écrit de «li eredi di Mengoccio de la Semola» (G., I, 2; «Nengoccio de la Semola», Z., 4v) $\rightarrow$ «les heritiers de Nengoce du Donts (I03r): ce qui nous intéresse ici n'est pas le Dont à la place de Son, car il s'agit sans doute d'une erreur dû à l'imprimeur, mais c'est le passage «Mengoccio» $\rightarrow$ «Nengoccio» $\rightarrow$ «Nengoce»; Mengoccio est en italien un prénom alors que «Neugoce» est clairement une adaptation phonologique de negozio, «magasin», faute qui confirme d'ailleurs la reconnaissance que le nom indiquait un travail précis, celui de vendre le son. ${ }^{36}$ Et c'est encore le cas de "Camillo Pocosale» (G., II, 3, Z., I4r; G., IV, 6, Z. 28v), qui devient "Camille» (II6r) et "Camille Poquesal» (I35r); l'omission aussi bien que le calque précis du nom montrent que le traducteur n'a pas saisi le caractère ironique de ce nom composé. Pocosale (poco sale, chez.Z.), qui joue évidemment sur l'adjectif poco, «peu», qui précise le modèle paradigmatique du substantif sale, "sel》), utilisé dans un sens figuré ("sagesse dans le raisonnement, dans l'action ou dans l'expression; intelligence, sapience») qui met en évidence la débilité mentale de Camillo et qui amplifie le portrait plaisant d'un type de personnage simple, comme l'était déjà le modèle du babbione, $d u$ gonzo ou $d u$ pecorone. ${ }^{37}$

La traduction française $d u$ Negromante apparaît ainsi comme un document important pour l'bistorien de la langue. Jean de La Taille a de temps en temps indiqué dans les marges de son texte les difficultés de traduction de certains mots: «aube neuve» et «chemise» (Iogr; et la variante Camiscia), choix de termes qui ne rend pas le jeu de mots italien càmice («un camicie / Nuovo», G., I, 3, Z., gr) et camìcie («camiscie», Z., gr), aussi bien que "pennaches», 
〈pots» et 〈pentole(s)» (Z., Iogr-v) pour traduire la déformation et la paronomasie pennacchi, pentacoli, pentole (G., I, 3; «pennachi», «pentaculo», «pentol(e)», Z., gr-gv). Nous voudrions, pour terminer, rappeler dans cette comédie de la magie l'importance du terme incanto $(G ., I, 3)$, pour lequel de La Taille utilise de manière variée «charme» (I08r), «ensorcellement» (I02v) et «malie» (Io8r, avec une glose explicative). ${ }^{38}$ Il faut partir du latin carmen (peut-être dérivé de canmen, forme de canere, «chanter»), formule religieuse aussi bien que magique, enchantement, qui est aussi présent en tant que verbe dans le latin post-classique carminare, «enchanter», et qui passe à l'ancien français charme (XII e siècle), avec sa référence originelle d'sinfluence magique, sortilège, enchantement); charmer se retrouve employé dans les dialectes italiens du Sud, certes à cause de la présence des Normands (à Naples 'nciarmë, incanto, stregoneria; dans la Calabre cermatu, fatato; dans la Sicile ciarmari, incantare, et ciarmu, incanto, malìa), dans ceux $d u$ Nord (par exemple dans le piémontais anciarmé, stregare, ou dans le milanais ingermá, incantare, ou dans le génois 'ngiarmá, stregato), et dans les dialectes du Centre (à Pérouse, 'ncialmé, incantare con le parole, dans le romanesque inciarmare, dans le «gallurese» de la Sardaigne incialmatu, incialmanatu, assorto, incantato). Le français «charme» indiquait en effet la formule rythmique utilisée pour produire un enchantement (des dérivés sont aujourd'bui: «charmer», "charmant», «charmerie», "charmeur», «charmeusement», «charmeux»), tout comme le latin carmen, d'où il provient. Ce n'est qu'au XVII siècle que ce mot exprimera la beauté féminine et son pouvoir magique sur les hommes, variation de sens qu'on constate en italien dans les mots incanto, incantare, incantevole, incantesimo (il faut mentionner l'histoire parallèle de fascino, qui à l'origine signifiait malocchio, «mawvais œil», «sort»). Cette évolution sémantique de carmen correspond d'autre part à la relation entre le charme de la parole prononcée et la magie de l'écriture, toujours selon le même procès qui conçoit dans ce terme latin une relation précise entre le vers chanté, la poésie, et la formule poétique douée d'une valeur magique. Exercer la connaissance de l'étymologie éclaire et stabilise le sens des mots; ainsi, sur ces exemples de passage d'une unité lexicale d'une langue dans une autre, par un cheminement souvent voilé, nous aurons, grâce à cette traduction qu'est Le Négromant de Jean de La Taille, une leçon caractéristique et un indice non moins vital et organisé d'un texte littéraire dont la langue est le reflet d'une culture.

Riccardo Benedettini 


\section{IL NEGROMANTE DE L'ARioste}

I. Voir T. Ambersley Daley, Jean de la Taille (I533-1608). Étude Historique et Littéraire, Paris, Librairie Universitaire J. Gamber, 1934, pp. 176-82. A propos du lien entre la politique et la poétique chez l'écrivain, voir E. Refini, «Profiter de quelque chose à ma république. Poetica e politica nell'epistola De l'art de la tragédie di Jean de La Taille», Studi francesi, I 58, 2009, LIII, pp. 234-50 Sur la traduction française des comédies de l'Arioste, voir Mariangela Miotti, «Le théâtre de l'Arioste en France. I Suppositì), in L'Arioste et le Tasse en France au XVI siècle, Paris, Éditions Rue d’Ulm, «Cahiers V. L. Saulnier», 20, 2003, pp. 99-II 8. Sur les comédies de l'Arioste possédées par la Fondation Barbier-Mueller, voir Poètes italiens de la Renaissance dans la Bibliothèque de la Fondation Barbier-Mueller. De Dante à Chiabrera, Catalogue établie par J. Balsamo, avec la collaboration de Fr. Tomasi, Genève, Droz, 2009, n. 29, pp. 69-70.

2. Toutes nos références sont celles de l'édition LE NEGROMANT, / COMEDIE DE M. LOUIS / ARIOSTE, NOUVELLEMENT MISE / en François, par Iehan de la / Taille de Bondaroy, in La Famine, ou les / GABEONITES, / Tragedie prise de la Bible, et / suivant celle de Saül. / Ensemble plusieurs autres Oeuvres poëtiques, de / IEHAN DE LA TAILLE de Bondaroy gentil- / homme du pays de Beauce, \& de feu Iaques de la / Taille son frere, desquels œuvres l'ordre se void en la / prochaine page. / (marque éditoriale: arbre avec devise en grec) / A PARIS. / Par Federic Morel Imprimeur du Roy. / M.D.LXXIII. / Avec Privilege dudit Seigneur. Nous avons consulté l'édition conservée à la Bibliothèque nationale de France (Tolbiac, RES-YE-1 81 8-1 822; feuillets numérotés 99vI42r). Le texte de cette édition, à laquelle renvoient les pages indiquées après les citations, a été respecté dans son détail; en vue d'une lecture plus aisée, nous avons seulement introduit l'opposition $u / v$, résolu les abréviations (par exemple: «hõme» pour «homme») ainsi que les esperluettes (\&). De cette traduction, nous avons également consulté: l'édition I 879, publiée à Genève par Slatkine Reprints en 1968, p. CVII-CCXXVII; JeAn De La TAILle, Le Négromant, Texte édité et présenté par Fr. Rigolot, in Théâtre français de la Renaissance, La comédie à l'époque d'Henri II et de Charles IX, Première Série, vol. 9 (I 566-I 573), Florence-Paris, Olschki-PUF, 1997, pp. I 27-207.

3. Voir Kathleen M. Hall, «A Defence of Jean de La Taille as a Translator of Ariosto», in The Modern Language Review, July 1972, Volume 67, Number 3, pp. 536-42.

4. Nous citons d'après Il Negromante in LE COMMEDIE di Ludovico Ariosto, sous la dir. de A. Gareffi, Volume II, Il Negromante, première rédaction, Il Negromante, deuxième rédaction, La Lena - I Studenti - La Scolastica - L'Imperfetta, Turin, UTET, 2007, pp. 439- 24 (sigle utilisé $G$.); nous indiquons les actes auxquels nous renvoyons en chiffres romains et les scènes en chiffres arabes. Sur l'histoire textuelle du Negromante, voir les deux «Note al testo» de A. Gareffi, op. cit., pp. 44I-43 et pp. 527-29. Voir aussi: Ludovico Ariosto, Commedie, sous la dir. de C. Segre, avec une introduction de L. Caretti, Turin, Einaudi, 1976 [1954 $]^{\mathrm{I}}$, travail critique qui prend comme texte de base l'édition de I $59 \mathrm{I}$; Ludovico Ariosto, Le Commedie, sous la dir. de M. Catalano, Vol. I, Bologne, Zanichelli, I933-XI, voir en particulier les pp. XXXIV-LI de l'Introduction ainsi que le Vol. II, Il Negromante (première rédaction), pp. I-92, et Il Negromante (deuxième rédaction), pp. 93-183.

5. De cette édition Zoppino de la Comedia di Messer Lodovico Ariosto nous avons consulté l'exemplaire conservé à la Bibliothèque Marucelliana de Florence: IL NEGROMANTE. / COMEDIA DI MES- / SER LODOVICO ARIOSTO. / (Portrait du Poète) / MDXXXV; explicit (fol. 35r): In Vinegia per Nicolo d'Ari- / stotile detto Zoppino. / M.D.XXXV (coll. R.u.ro; 36 feuillets non numérotés, in- $8^{\circ}$ petit format; sigle utilisé $Z$ ). Le lecteur dispose, dans ce volume, d'autres œuvres de l'Arioste publiées par Zoppino: Le Rime ( I 546), Le Satire (1 535), La Lena (1 535) et I Suppositi ( I 525 ). Nous avons suivi une orientation éminemment conservatrice, aussi bien pour la ponctuation que pour l'accentuation, qui sont très différentes du système moderne, mais qui peuvent offrir un témoignage significatif aux spécialistes d'histoire de la langue. Nous remercions Mmes Maria Prunai Falciani et Rossella Todros pour leur aide dans nos recherches à la Marucelliana. Sur Niccolò di Aristotile detto 


\section{RicCARDO BENEDETTINI}

Zoppino, l'éditeur des trois premières éditions des livrets luthériens publiés «sous le masque d'Erasme», voir Rosanna Gorris, Alla corte del principe. Traduzione, roman₹o, alcbimia, scienza e politica tra Italia e Francia nel Rinascimento. Préface di Jean Balsamo, Annali dell'Università di Ferrara (Nuova Serie), Sezione VI Lettere, vol. IX, n. I, Università degli Studi di Ferrara, 1996, p. 36; de Rosanna Gorris, voir aussi les «Conclusions» détaillées au volume L'Arioste et le Tasse en France an XVI $T^{e}$ siècle, op. cit., pp. 257-74.

6. A. Cioranescu, L'Arioste en France. Des origines à la fin du XVII siècle, tome I, Paris, Les Éditions des Presses Modernes, I939, pp. 30 I-307. Mêmes formulations chez T. Ambersley Daley, Jean de la Taille, op. cit., p. I77. Voir aussi «Jean et Jacques de La Taille», in É. Chasles, La comédie en France au seizième siècle, Genève, Slatkine Reprints, 1969, pp. 87-102. Sur l'attraction française pour le monde poétique de l'Arioste, voir: Chiara Lastraioli, «Arioste en France au xvI ${ }^{\mathrm{e}}$ siècle», in Dictionnaire des lettres françaises. Le XVI siècle, éd. revue par M. Simonin, Paris, Fayard, 200I, pp. 66-68; J. Balsamo, «L'Arioste et le Tasse. Des poètes italiens, leurs libraires et leurs lecteurs français», in L'Arioste et le Tasse en France an XVI siècle, op. cit., pp. I I-26; E. Balmas, «Note sulla fortuna dell'Ariosto in Francia nel Cinquecento», in Le prime tradurioni dell'A Ariosto, Atti del V Convegno sui problemi della traduzione letteraria (Monselice, 27 giugno 1976), Padoue, Editrice Antenore, 1977, pp. 3-32; E. Bottasso, «Le commedie di Ludovico Ariosto nel Teatro francese del Cinquecento», Giornale storico della letteratura italiana, CXXVIII, I95 I, p. 4I-80: en particulier, pp. 5 I-65.

7. M. Catalano a souligné que le premier Negromante utilisait une langue plus franche et impure que le second, qui, à son avis, a perdu une partie de sa fraîcheur d'origine. Voir M. Catalano, Vita di Ludovico Ariosto. Ricostruita su nuovi documenti, Vol. I, Genève, Olschki, S.A., Éditeur, I930, p. 380 et suivantes. Pour les variantes du texte italien, voir Il Negromante, éd. Angela Casella, in Tutte le opere di Ludovico Ariosto, sous la dir. de C. Segre, Volume IV, Commedie, publiées par Angela Casella, Gabriella Ronchi et Elena Varasi, Milan, Mondadori, I 974, en particulier pp. 922-48.

8. Les citations de l'italien dans les marges de la traduction constituent un sujet de grand intérêt pour la recherche. Sur la question de la 'reproduction' de l'italien dans le français du $\mathrm{XVI}^{\mathrm{e}}$ siècle, on renverra le lecteur aux études sur les traductions de Larivey par Anna Maria Raugei, travaux centrés sur des textes spécifiques, mais qui en arrivent à des conclusions d'une importance générale: «Un esempio di comicità verbale: il 'jargon' pedantesco nelle commedie di Pierre de Larivey», in Saggi e e ricerche sul teatro francese del Cinquecento, Florence, Olschki, I985, pp. I4I-66, et «Italianismo e italianismi nel teatro di Pierre de Larivey», in La letteratura e l'immaginario. Problemi di semantica e di storia del lessico franco-italiano, Atti dell'XI Convegno della Società Universitaria per gli studi di lingua e letteratura francese, Verona I4-I6 ottobre I982, pp. 40I-2 I. Sur la traduction de l'italien, voir J. Balsamo, «Traduire de l'italien. Ambitions sociales et contraintes éditoriales à la fin du XvI ${ }^{\mathrm{e}}$ siècle», in Traduire et adapter à la Renaissance, Actes de la journée d'étude organisée par l'École nationale des chartes et le Centre de recherche sur l'Espagne des XVI ${ }^{\mathrm{e}}$ et XVII ${ }^{\mathrm{e}}$ siècles (Paris, I I avril I 996 ) réunis par Dominique de Courcelles, Paris, École des Chartes, 1998, pp. 89-98.

9. Sur certains italianismes du Négromant, voir Fr. Rigolot, op. cit., p. I 34-135. Sur cette tendance à l'italianisme au $\mathrm{xvI}^{\mathrm{e}}$ siècle, voir: Les traductions de l'italien en français $d u X V T^{e}$ au $X X^{e}$ siècle, Actes du Colloque International Monopoli, 4-5 octobre 2003, par G. Dotoli, Fasano, Schena Editore, Paris, Presses de l'Université de Paris-Sorbonne, 2004; J. Balsamo, Rencontres des Muses. Italianisme et anti-italianisme dans les lettres françaises à la fin du XVI siècle, Genève, Slatkine, 1992; voir également É. Picot: Des français qui ont écrit en italien au XVI ${ }^{e}$ siècle, Paris, Bouillon, 1902; Les français italianisants an XVT siècle, Paris, Champion, 19061907, 2 vol.; Les Italiens en France au XVI siècle, Introduction de N. Ordine, Manziana (Rome), Vecchiarelli editore, i995 [réimpression anastatique de l'édition, Bordeaux 1918]. Voir aussi «La comédie italienne en France au xvI ${ }^{\mathrm{e}}$ siècle», in R. Lebègue, Études sur le théâtre 


\section{IL NEGROMANTE DE L'Arioste}

français, I, Moyen Âge, Renaissance, Baroque, Paris, Éditions A.-G. Nizet, I977, pp. 277-96: en particulier, pour cette traduction, p. 28I.

ı. Voir A. De Luca, «I prologhi delle commedie ariostesche», in Atti dei Convegni Lincei 6, Convegno Internazionale Ludovico Ariosto, Roma-Lucca-Castelnuovo di GarfagnanaReggio Emilia-Ferrara (27 settembre-5 ottobre 1974), Rome, Accademia Nazionale dei Lincei, I975, pp. 577-89, en particulier p. 580 et suivantes.

I I. Ces thèmes, repris de l'Ars Poetica d'Horace (v. 39I et suivants), sont réélaborés par l'Arioste dans sa Satira VI. Voir Ludovico Ariosto, Satire e Lettere, sous la dir. de C. Segre, introduction de L. Caretti, Turin, Einaudi, 1976 [1954 $]^{1}$, p. 70.

I 2. Les termes savants «Fantesque», «Fizicien» et «Negromant» sont des emprunts que le français fait ici à l'italien. À propos de la perte de certains italianismes au cours du développement de la langue française, voir Ed. Huguet, Mots disparus ou vieillis depuis le XVI ${ }^{e}$ siècle, Paris, Droz, 1935, p. I30. Pour l'étymologie et l'utilisation de ces mots en italien, voir Fantésca, Fisico et Negromante in Grande Dižionario della lingua italiana, fondé par S. Battaglia, sous la dir. de G. Bàrberi Squarotti, Turin, UTET, I96I-2002, ad voces, [= BATTAgLIA; suivi par l'indication du volume et des pages], respectivement: V, 656; VI, 3I; XI, 334. Le «negromante», personnage nouveau chez l'Arioste, est un imposteur qui s'appelle Lachelino (G., II, $2 \rightarrow$ I I 4v). Il s'agit d'un juif de la Castille qui pratique les arts de l'escroquerie: l'alchimie, l'évocation des esprits, la médecine (Fisico signifie médecin). Une fois à Crémone, il s'improvise «negromante». Au Negromante de l'Arioste (figure qui dérive du «negromante» Ruffo de la Calandria et du faux médecin Callimaco de la Mandragola), s'inspirent la Strega de Lasca et l'Astrologo de Della Porta. Les nombreuses éditions du Negromante, ses influences intertextuelles et ses traductions (il faut rappeler aussi la traduction en prose latine effectuée par Juan Pérez) montrent un succès littéraire, plutôt que théâtral, qui est évidemment lié au Furioso.

I3. Rappelons par exemple qu'Aurélie et Marguerite se tutoient, que Cinthien tutoie Themole, le Fizicien, le Nebbie, la Mère, et la Fantesque; en revanche, les couples Lippe / Cambien et Fizicien / Maxime se vouvoient.

I4. Nous signalons les omissions entre parenthèses [] et les variations en caractères italiques. Nous donnons le texte de l'Arioste le plus vulgarisé (G.) suivi du texte varié de $Z$. et de la traduction française.

I 5 . Voir P. Zumthor, La lettre et la voix. De la 'littérature' médievale, Paris, Seuil, 1987.

I6. En italien il faut souligner aussi l'homophonie entre le nom du personnage Cambio et le substantif «cambio», jeu de mots qui se perd dans le passage au français de cette expression prononcée par Lippe, «n'abbi il cambio» (I, 2; «n’habbi il cambio», Z., 4r) $\rightarrow$ «vous aurez de moy la pareille» (Io4r). De même pour cette idée de «mutation» qui est implicite dans les mots de Cambien, «Non temo che si muti» (I, 2; $Z$., 8r) $\rightarrow$ «Ie n'ay pas peur qu'il se change» (I07r). Sur certains aspects de la langue utilisée par l'Arioste dans cette comédie, voir Maria Luisa Doglio, «Lingua e struttura del Negromante», in Ludovico Ariosto: lingua, stile e tradizione, Atti del Congresso organizzato dai comuni di Reggio Emilia e Ferrara I 2-I6 ottobre 1974, sous la dir. de C. Segre, Milan, Feltrinelli, 1976, pp. 427-43.

17. Il va de soi que cette traduction française respecte la conclusion de la première rédaction du Negromante, conclusion qui est bien différente de celle, beaucoup plus connue (et représentée), de I 5 I. Pour une analyse des variantes de la mise en scène de cette comédie, non seulement du point de vue de l'harmonisation des niveaux textuels, voir Angela Guidotti, Il modello e la trasgressione: commedie del primo '500, Rome, Bulzoni editore, I983, p. 38 et suivantes. 


\section{RicCARdo BENEDETTINI}

I 8. Dans l'éd. I879, le texte se termine par des points de suspension (CXXXVIIII), ce qui nous semble confirmer cette volonté d'adaptation plutôt qu'une lecture erronée de l'original.

I9. En marge du texte français, nous lisons la réplique italienne «Che nasca / una fistola». Rappelons que Léon X souffrait de fistules. Le terme «fistola» comporte évidemment une allusion vulgaire. Voir à ce propos Luigia Zilli, «La commedia 'Gl'Ingannati' e la sua traduzione francese: Due comicità a confronto», in Studi di letteratura francese, $X$, Commedia e comicità nel Cinquecento francese e europeo, Florence, Olschki, MCMLXXXIII, pp. 3 I-5 I: p. 48. Il convient de souligner que les prénoms italiens Aurelia et Emilia sont des allusions aux anciennes rues romaines, tout comme le prénom Lavinia qui renvoie à un personnage de la mythologie romaine.

20. Nouvelle variante de la geste érotique dans l'éd. de i 5 I: «Deh, non temer che giostrino, / che la lancia è spuntata e trista e debole».

2 I. Indispost «adj. indisposé, faible», in Fr. Godefroy, Lexique de l'ancien français, Paris/Leipzig, H. Welter éditeur, I90 I, p. 286.

22. Les nombres, qui dans cette comédie sont utilisés pour des renseignements sur le temps, sur l'argent à payer au Negromante, mais surtout pour confondre l'interlocuteur, constituent un lieu de variation fréquente: «tre, sei mesi, nove o dodici» $(G ., I, 2) \rightarrow$ «deux mois, six, neuf ou dix» (Io7r); «dodici lire e mezo» (G., I, 3) $\rightarrow$ «cent solz» (I०9v); «undici» $(G ., \mathrm{I}, 3) \rightarrow$ «quatre francz et demi» (Iogv); «sedici / mesi» $(G .$, II, 5) $\rightarrow$ «treize mois» (I 2or); «fa che tu mi truovi otto e otto sedici, / et otto ventiquattro, e appresso quindici / e quindici fa trenta, et altri quindici / quarantacinque (sono, se ben numero, / settantré) fiorini» (G., III, 2) $\rightarrow$ «faites que vous me trouviez, huit et huit, seize, et huit, vint quatre, et apres quinze, ce sont quarante cinq, si ie compte bien, septante quatre florins» (I 22v): il s'agit de l'argent demandé par le Fizicien, question réargumentée par Cinthien avec «Settantré fiorini ci bisognano, / e non manco ?» $\rightarrow$ «Fault il bien septante trois florins, et non moins» (I 23r); «Or vedi anche di giungerci / altri ventitré appresso» (G., III, 2) $\rightarrow$ «Or voy encor de XV autres et XXIII apres» (I 23r). Sur les possibles motivations de ces écarts dans une traduction du $\mathrm{XVI}^{\mathrm{e}}$ siècle, on trouvera des indications dans notre article «Il De la Naissance, duree et cheute des estats da René de Lucinge a Girolamo Naselli. Osservazioni sulle modalità di traduzione», Pubblicazioni dell’Università di Bari, Annali della Facoltà di Lingue e Letterature Straniere, terza serie / 2006-2007 / XVIII, Schena editore, pp. 409-23: p. 4I9.

23. Bouc. Mari trompé, I, $6_{3} 6$ in Ed. Huguet, Dictionnaire de la langue française du seizième siècle, Paris, Didier, I925-1967, ad voces, [= Huguet; suivi par l'indication du volume et des pages]. Voir aussi Cocu: «ce nom estant actif et passif, nous disons Cocu cocuant, et Cocu cocué», ivi, II, 325 . Sur l'étymologie très obscure du terme «cocu», voir P. Guiraud, Les gros mots, Paris, Puf, coll. «Que sais-je ?», pp. 7I-73, selon lequel «cocu» ne viendrait pas de cuculus, le «coucou», oiseau dont la femelle pond ses œufs dans un nid étranger, mais de cucullus, «capuchon» jaune, du narcisse des prés et de la primevère officinale, qui métaphoriquement rend l'homme trompé, le «cocu», «aveugle» et «réduit à l'impuissance». Voir aussi les mots Becco et Cappiuccio, in Battaglia, II, p. I 40 et p. 724-725.

24. On trouve la césure de domine dans un autre passage du texte: Cambio. Ove [domine] / corre costui ? (G., IV, I; Z., 25v) $\rightarrow$ CAmbien. Ou court cestuy ? (CXCIV). Voir Dòmine, in Battaglia, IV, p. 937-938.

25. Battaglia, Babbióne (babióne), I, 919. Il faut signaler la forme diminutive «enfançon» qui sera remplacée par «petit enfant». Voir Ed. Huguet, Mots disparus..., op. cit. Voir aussi l'expression «Deh, pecorone» $(G ., \mathrm{XXX}) \rightarrow$ «Beste que tu es» (XXXXX), où pecorone (à la lettre, 


\section{IL NEGROMANTE DE L'Arioste}

«grand bouc»), synonyme de scimunito, «idiot», est reconduit à son hyperonyme. Sur ce terme voir encore Luigia Zilli, art. cit., p. 48.

26. Battaglia: Cinghiale, III, p. i 5 , et ses variantes anciennes et régionales Zingiale (XXI, p. I०80), Zingiaro (ibidem, terme employé au Nord, avec changement de suffixe); Zingaro (Zingherlo, Zinghero, ibidem), l'ancienne forme Cingaro (III, p. I 53 ; à rappeler le mot Cingano, terme rare et littéraire qui signifie joueur de tzigane, III, p. I 53), Gitano (VI, p. 855), «bohémien espagnol», mot emprunté à l'espagnol gitano, du lat. Aegyptanus (lat. class. aegyptianus), de Aegyptus, Egitto, «Égypte» (en fr. «gitan» est attesté depuis I 570), car on croyait que les gitans étaient venus de l'Égypte (Égyptien, aphérèse d'Egitano).

27. Battaglia : Gónzo, VI, p. 982: l'étymologie du mot est incertaine; on a conjecturé une dérivation par aphérèse du lat. [vere]cundius, vergognoso, ritroso (avec lénition du son guttural dans le Nord de l'Italie), de verecundia, vergogna, pudore, ritrosia.

28. Il faut souligner que le texte de l'Arioste change dans l'édition de I55 I: Astr. [...] Ma eccolo. / Sta pure all'erta, e fa il grossieri, e mostrati / di non aver le capre. / Nib. Starò tacito. Le changement lexical n'implique pas une variation au niveau de la signification du texte: Grossière (grossièro, grossèro, grossièr), in BATTAGLIA, VII, p. 65: fare il grossiere équivaut à fare il tonto (l'exemple est tiré de l'édition de I 5 I I de cette comédie); grossière, du provençal «grossier» (issu de gros, grossus, qui en latin de basse époque a remplacé crassus, d'où est issu gras), adjectif répandu dans le dialecte piémontais (en français, "grossien», depuis I 599 ). L'expression non aver(e) le capre signifie non sapere niente (Battaglia: Capra, II, pp 725-26), «ne savoir rien».

29. Voir l'expression «charger quelqu'un de bois», Ed. Huguet, Le langage figuré ..., op. cit., p. 215.

30. Voir Ciància, in Battaglia, III, p. Io8. D'où chez de la Taille des expressions comme «babiller» (I, 4, CXXXVIII), «songes et follie» (II, I, CXLIV).

3i. Sur le gouvernement de la Garfagnana, voir Ludovico Ariosto, Lettere, éd. A. Stella, Milan, Mondadori, I965, en particulier l'Introduction, p. V-XXVIII. Sur la Garfagnana et le Negromante, voir aussi H. Hauvette, L'Arioste et la poésie chevaleresque à Ferrare au début du $X V T^{e}$ siècle, Paris, Champion, 1927, en particulier p. I 27 et suivantes.

32. L'allusion au vol est davantage accentuée dans la seconde rédaction du Negromante, où on lit Graffignana (G., vers I 304).

33. Eleganżia exprime la langue de la cour, qui est ici indiquée comme modèle, et qui se rapproche du choix de la langue du Bembo, choix que l'Arioste va définir à cette période. Lombarda est la langue maternelle de l'Arioste, c'est-à-dire la langue padana, celle qui est parlée dans la plaine du Pô.

34. Sur le très célèbre Studio de Padoue, voir B. Brugi, «Gli antichi scolari di Francia allo Studio di Padova», in Mélanges offerts à M. Picot par ses amis et ses élèves, (Réimpression de l'édition de Paris, I9I 3), Slatkine Reprints, Genève, 1969, tome I ${ }^{\mathrm{er}}$, pp. 535-55. Sur un moment important de cet univers culturel francophile, voir Anna Bettoni, «La francofilia di Pietro Buccio: un accademico bresciano a Padova nel Cinquecento», in Incroci. Scritti in onore di Mario Mammi, sous la dir. de R. Dell'Acqua, Cr. La Rocca, G. Zanotti, Padoue, CLEUP, 2006, pp. 17-31.

35. Une autre indication est «osteria del Bufolo» (G., IV, 2; «hostaria del Buffolo», Z., 26r) $\rightarrow$ «hostellerie du Buffle» (CXCVII), ou buf(f)olo, variante de bufalo, équivaut ici à sciocco, «sot», «simple». Ces renseignements sur la route à suivre, comme on pouvait déjà le remar- 


\section{Riccardo BenedetTini}

quer pour les nombres, ne sont que des jeux qui visent à créer une confusion chez le récepteur du discours, pour le tromper ou pour le convaincre d'une fausse vérité.

36. Mengoccio, Copo, Nanna sont des prénoms, plutôt que des vrais personnages, qui ne font que compliquer le cours de l'action.

37. Voir Battaglia, Sale, XVII, p. 395. Le composé pocosale est inventé sur les modèles: pocobòno, pocodibuòno, pocopotènte, pocotenènte (ivi, XIII, pp. 697-98). L'origine du nom est expliquée dans la seconde rédaction du texte, «(Quando lo battezzâr non doveva essere / Sale al mondo, che non trovâr da porgliene / Un grano in bocca.)», vv. 755-57, et, comme l'a souligné Angela Casella, ce personnage dérive du Calandro de Bibbiena, qui est à la fois créé sur le modèle de Calandrino de Boccaccio («Presentazione», in op. cit., p. XXVIII).

38. Voir in Battaglia les mots: Carme, II, p. 775; Incanto, VII, pp. 6io-i i; Malia, IX, pp. 542-43; Sòrte, XIX, pp. 507-IO. 